\title{
ARTICLE OPEN \\ Synthesis of ultrathin two-dimensional nanosheets and van der Waals heterostructures from non-layered $\gamma$-CuI
}

\author{
Kangkang Yao ${ }^{1}$, Peng Chen ${ }^{1,2}$, Zhengwei Zhang ${ }^{1}$, Jia $\mathrm{Li}^{1}$, Ruoqi $\mathrm{Ai}^{1}$, Huifang $\mathrm{Ma}^{1}$, Bei Zhao ${ }^{1}$, Guangzhuang Sun ${ }^{1}$, Ruixia Wu ${ }^{1}$,
} Xuwan Tang ${ }^{1}$, Bo $\mathrm{Li}^{3}$, Jiawen $\mathrm{Hu}^{1}$, Xidong Duan ${ }^{1}$ and Xiangfeng Duan ${ }^{2}$

Two-dimensional (2D) nanosheets have attracted considerable recent interest for their atomically thin geometry and unique thickness-dependent electronic properties. The 2D nanosheets studied to date are generally limited to intrinsically layered materials, in which the covalently bonded atomic layers are held together by weak van der Waals forces and can be readily exfoliated to single or few-atom thick nanosheets. To prepare 2D nanosheets from non-layered materials can greatly expand the scope of 2D materials, but is much less straightforward. Here, we report the successful synthesis of ultrathin nanosheets from nonlayered $\gamma$-Cul on $\mathrm{SiO}_{2} / \mathrm{Si}$ substrate using a facile physical vapor deposition process. The resulting $\gamma$-Cul nanosheets display a triangular and hexagonal geometry with the lateral dimension up to $5 \mu \mathrm{m}$ and thickness down to $1 \mathrm{~nm}$. Raman spectroscopy, X-ray diffraction, and transmission electron microscopy studies demonstrate the resulting nanosheets retain single-crystalline $\gamma$-Cul phase. Additionally, we further show the $\gamma$-Cul nanosheets can be readily grown on other $2 \mathrm{D}$ materials $\left(e .9 ., 2 \mathrm{D}-\mathrm{WSe}_{2}, 2 \mathrm{D}-\mathrm{WS}_{2}\right)$ to form van der Waals heterostructures (vdWHs). Optical microscopy images and Raman intensity mappings confirm the formation of $\gamma$-Cul/WS $\mathrm{WS}_{2}$ and $\mathrm{C}$-Cul/WSe $\mathrm{Wertical}_{2}$ heterostructures. The electrical transport studies show that $\mathrm{\gamma}$-Cul nanosheets exhibit a low resistivity of $\sim 0.3 \Omega \mathrm{cm}$ and $\gamma$-Cul/WS 2 vertical heterostructures display a $p-n$ diode behavior with distinct current rectification. The synthesis of $\mathrm{\gamma}$-Cul nanosheets and heterostructures open a pathway to ultrathin nanosheets and van der Waals heterostructures from non-layered materials and could open up exciting opportunities in electronics and optoelectronics.

npj 2D Materials and Applications (2018)2:16; doi:10.1038/s41699-018-0058-2

\section{INTRODUCTION}

Two-dimensional (2D) materials have attracted significant attention as a new generation of atomically thin material for nextgeneration electronic and optoelectronic devices due to their ultrathin geometry and unique thickness-dependent physical properties. In the past decade, significant efforts have been devoted to synthesizing graphene ${ }^{1}$ and graphene-like 2D materials including hexagonal $\mathrm{BN}_{1}{ }^{2}$ transition metal dichalcogenides (TMDs) (such as $\mathrm{MoS}_{2}{ }^{3} \mathrm{WS}_{2}{ }^{4}$ and $\mathrm{WSe}_{2}{ }^{5}$ ), metal oxides (such as $\mathrm{MoO}_{3}{ }^{6} \mathrm{WO}_{3}{ }^{7}$ ), and metal halides (such as $\mathrm{Pbl}_{2}{ }^{8} \mathrm{Cdl}_{2},{ }^{9} \mathrm{Bil}_{3}{ }^{10}$ and $\mathrm{Crl}_{3}{ }^{11}$ ) using various approaches. These $2 \mathrm{D}$ materials typically feature unconventional physical properties distinct from their bulk crystals, which can open up exciting opportunities for both the fundamental investigation of low dimensional chemistry and physics and potential technological applications at the limits of single atomic thickness. ${ }^{12-21}$ Some available 2D materials may also be flexibly combined to produce new van der Waals heterostructures (vdWHs) with atomic sharp modulation of chemical composition and electronic structure. Such vdWHs can enable the creation of tailored heterojunctions without the traditional lattice matching requirement, and thus offer a much more flexible approach for heterogeneous material integration than the traditional semiconductor heterostructures. It can thus open up a limitless possibility to nearly arbitrarily combine and control different properties, and develop exciting new technologies beyond the reach of existing materials. Recent studies have already demonstrated the exciting potentials of 2D materials and vdWHs in nano-electronics applications, such as atomically thin transistors, ${ }^{22,23}$ vertical field-effect transistors, ${ }^{24}$ and optoelectronics applications, such as photodetectors ${ }^{25}$ and light-emitting diodes. $^{26,27}$

Although it has been relatively straightforward to produce ultrathin 2D nanosheets from intrinsically layered materials using various synthetic approaches, it is considerably more difficult to grow highly anisotropic ultrathin nanosheets from non-layered materials due to their intrinsic three-dimensional lattice structure. Nonetheless, a few examples of 2D nanosheets of some nonlayered materials (such as $\mathrm{ZnSe}^{28} \mathrm{PbS}^{29}$ ) and heterostructures (such as $\mathrm{CdS} / \mathrm{MoS}_{2}{ }^{30} \mathrm{PbS} / \mathrm{MoS}_{2}{ }^{31}$ ) have been reported recently. Copper iodide (Cul), as a non-layered I-VII group compound, crystallizes into three different phases: $a, \beta$, and $\gamma$ and shows different crystal lattices with increasing temperature. Cul undergoes a phase transition from the cubic $\gamma$-phase to the hexagonal $\beta$-phase above $369^{\circ} \mathrm{C}$. The $\beta$-phase can be further transformed to the cubic $\alpha$-phase with increasing temperature above $407^{\circ} \mathrm{C} .{ }^{32}$ Figure $1 \mathrm{a}, \mathrm{b}$ is the stick-and-ball crystal structure model of $\mathrm{\gamma}$-Cul. The low-temperature $y$-phase Cul crystal is a p-type semiconduc$\operatorname{tor}^{33}$ and has a cubic zinc blende structure with the space group F$43 \mathrm{~m}$. In the crystal of $\mathrm{Y}$-Cul, each $\mathrm{Cu}^{+}$ion is tetrahedrally surrounded by four $\mathrm{I}^{-}$ions (Fig. 1a). Currently, $\mathrm{Y}$-Cul has been

\footnotetext{
${ }^{1}$ State Key Laboratory for Chemo/Biosensing and Chemometrics, College of Chemistry and Chemical Engineering, Hunan University, 410082 Changsha, China; ${ }^{2}$ Department of

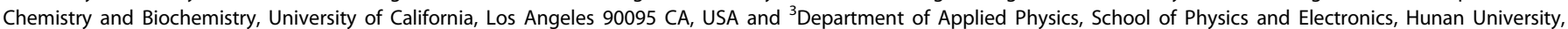
410082 Changsha, China

Correspondence: Jiawen Hu (jwhu@hnu.edu.cn) or Xidong Duan (xidongduan@hnu.edu.cn) or Xiangfeng Duan (xduan@chem.ucla.edu)
}

Received: 18 January 2018 Revised: 12 April 2018 Accepted: 16 April 2018 
extensively applied in fields such as organic catalysts, ${ }^{34}$ structural template of organic semiconductor, ${ }^{35}$ solid state dye-sensitized solar cells $s^{36,37}$ and hole conductor in perovskite solar cells. ${ }^{38,39}$

The preparations of $y$-Cul nanoparticles, ${ }^{40}$ nanowires, ${ }^{41}$ thin films ${ }^{42}$ have been studied using either physical or chemical methods. Furthermore, there are several reports on the synthesis of the $\mathrm{\gamma}$-Cul single crystal nanoplates with the thickness of $60-90 \mathrm{~nm}$ via PEG/TSA-assisted solution-based methods. ${ }^{43,44}$ However, the synthesis of regular and ultrathin $\gamma$-Cul nanosheets has not been reported. Here, we report a facile physical vapor deposition method to prepare high-quality single-crystalline $\gamma$-Cul nanosheets with the thickness down to $1 \mathrm{~nm}$. Optical microscopy $(\mathrm{OM})$, atomic force microscopy (AFM) and scanning electron microscopy (SEM) studies show triangular and hexagonal

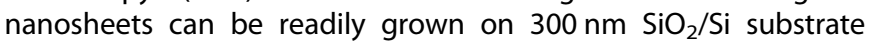
with the lateral dimension up to $5 \mu \mathrm{m}$ and thicknesses down to $1 \mathrm{~nm}$. Raman spectroscopy studies of the nanosheets reveal the transverse-optical (TO) mode of $\gamma$-Cul at around $122 \mathrm{~cm}^{-1}$. X-ray diffraction (XRD) studies show highly crystalline nature of the obtained $\gamma$-Cul samples with cubic phase. Energy-dispersive X-ray spectroscopy (EDX) studies reveal that the atomic ratio between $\mathrm{Cu}$ and $\mathrm{I}$ is about 1:1. Transmission electron microscopy (TEM) and the corresponding selected area electron diffraction (SAED) studies demonstrate that the $\gamma$-Cul nanosheets are single crystalline. Take a step further, we also show that the $\gamma$-Cul nanosheets can also be readily grown on typical $2 \mathrm{D}$ materials to form $\mathrm{\gamma}$-Cul/
WSe $e_{2}$ and $\mathrm{\gamma}$-Cul/WS $\mathrm{WS}_{2}$ van der Waals vertical heterostructures (vdWHs). The current-voltage curves show that $\gamma$-Cul nanosheets have low resistivity and $\gamma-\mathrm{Cul} / \mathrm{WS}_{2}$ vertical heterostructure show $\mathrm{p}-\mathrm{n}$ diode behavior with distinct current rectification behavior.

\section{RESULTS AND DISCUSSION}

The $\mathrm{\gamma}$-Cul nanosheets were synthesized on $\mathrm{SiO}_{2} / \mathrm{Si}$ substrate using a home-built physical vapor deposition (PVD) system (Figure S1), with $\gamma$-Cul powder as the precursor and argon as the carrier gas. The as-synthesized $\gamma$-Cul nanosheets typically display a triangle shape and occasionally a hexagonal shape with lateral size in the range of $1-5 \mu \mathrm{m}$ (Fig. 2a, Figure S2). The different $\gamma$-Cul nanosheets show highly distinct colors, which can be attributed to variable optical interference resulted from the different thicknesses. In addition to the relatively thick nanosheets with obvious contrast to the substrate color, there are a large number of triangular domains with rather weak contrast (highlighted by white arrows in Fig. 2a). The thickness of nanosheets ranges from $300 \mathrm{~nm}$ to $1 \mathrm{~nm}$ (Figure S2, Fig. 2b). Figure 2b further shows a high resolution $\mathrm{OM}$ image of an ultrathin $\mathrm{Y}$-Cul nanosheet grown on the $300 \mathrm{~nm} \mathrm{SiO} / 2$ Si substrate. The triangle nanosheet shows nearly the identical color with substrate due to its ultrathin thickness. The thickness can be further characterized using AFM. Figure 2c reveals the corresponding AFM image where the nanosheet has a
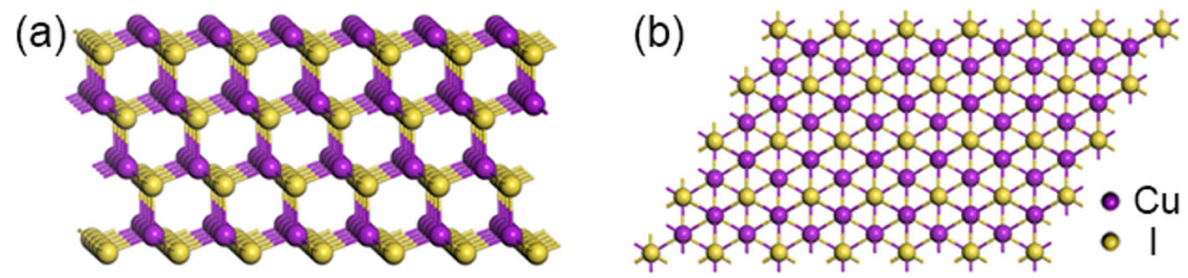

Fig. 1 Stick-and-ball crystal structures of $\gamma$-Cul. a Side view of the cubic crystal structure of $\gamma$-Cul along the (111) plane. $\mathbf{b}$ Top view of $\gamma$-Cul cubic crystal above the (111) plane. Purple and yellow spheres correspond to copper and iodine, respectively
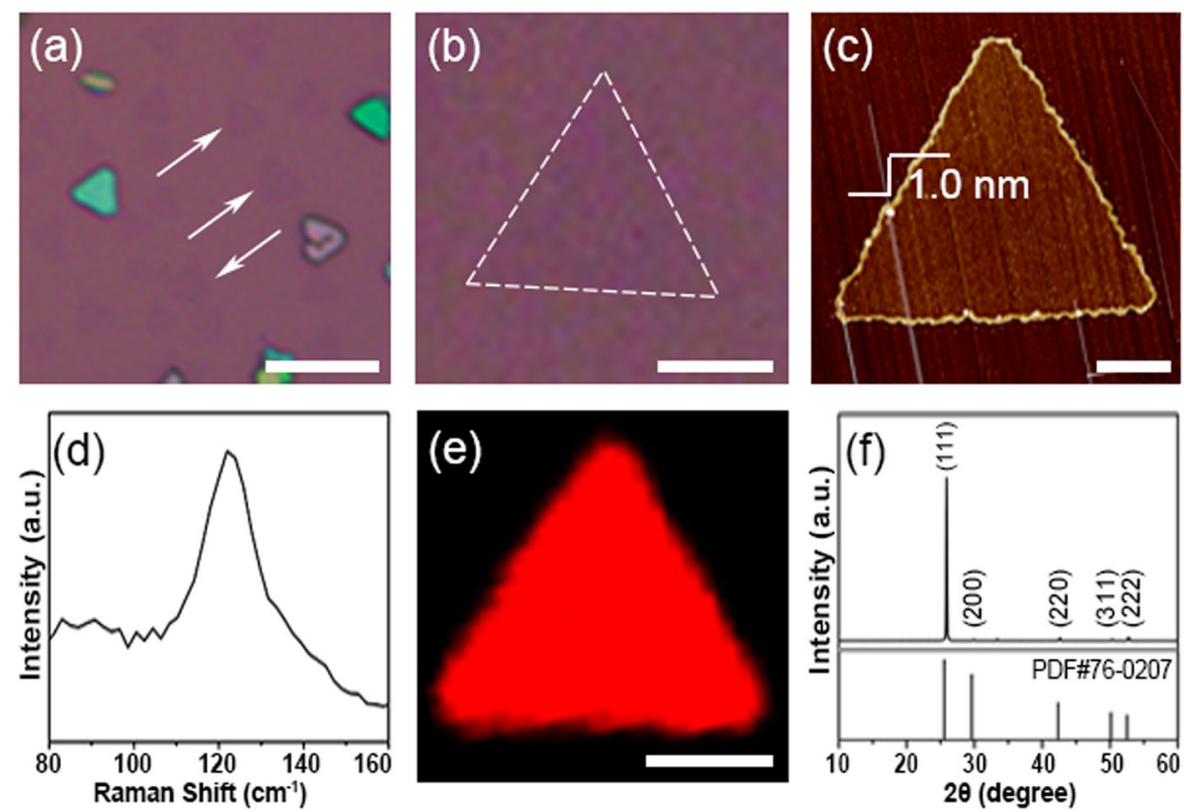

Fig. 2 The basic characterizations of $\gamma$-Cul nanosheets. a The OM image of the $\gamma$-Cul nanosheets grown on the $\mathrm{SiO}_{2} / \mathrm{Si}$ substrate; The white arrows highlight the ultrathin nanosheets that are barely visible. Scale bar, $10 \mu \mathrm{m}$. b, c The OM image and AFM image of an ultrathin $\gamma$-Cul nanosheet. Scale bar, 2, $1 \mu \mathrm{m}$, respectively. d Raman spectrum of the $\gamma$-Cul nanosheets. Raman experiment was performed in a confocal spectrograph using a $532 \mathrm{~nm}$ excitation laser. e The Raman intensity mapping of a typical $\gamma$-Cul nanosheet with Raman peak located at $122 \mathrm{~cm}^{-1}$. Scale bar, $2 \mu \mathrm{m}$. $\mathrm{f}$ XRD pattern of $\gamma$-Cul nanosheets grown on the $\mathrm{SiO}_{2} / \mathrm{Si}$ substrate 



Fig. 3 SEM and TEM characterizations of $\gamma$-Cul nanosheets. a SEM image of triangular $\gamma$-Cul nanosheets grown on $\mathrm{SiO}_{2} / \mathrm{Si}$ substrate; Scale bar, $1 \mu \mathrm{m}$. b SEM-EDX profile for the obtained $\gamma$-Cul nanosheets. c LR-TEM image of a typical triangle $\gamma$-Cul nanosheet supported on a grid of copper; Scale bar, $50 \mathrm{~nm}$. d HR-TEM image of a $\gamma$-Cul nanosheet, the inset is the corresponding SAED pattern of the nanosheet; Scale bar, $2 \mathrm{~nm}$, the scale bar in the inset is $21 / \mathrm{nm}$

thickness of $\sim 1 \mathrm{~nm}$, indicating the formation of ultrathin $2 \mathrm{D}$ structure.

We have next conducted Raman and XRD studies on the resulted $\gamma$-Cul nanosheets (Fig. 2d-f). The Raman spectra of the resulted $\gamma$-Cul nanosheets exhibits a prominent characteristic peak around $122 \mathrm{~cm}^{-1}$ when excited with a $532 \mathrm{~nm}$ laser (Fig. $2 \mathrm{~d}$ ), in agreement with the TO mode of bulk $\gamma$-Cul at room temperature. ${ }^{45}$ We were not able to observe the longitudinal-optical (LO) mode at $140 \mathrm{~cm}^{-1}$, which can be interpreted by the reason that the LO peak may be sub-merged in the broad TO peak resulting from the existence of the disorder in the structure of $\mathrm{y}$-Cul. ${ }^{46} \mathrm{~A}$ spatially resolved mapping of the Raman signal $\left(122 \mathrm{~cm}^{-1}\right)$ shows highly uniform contrast throughout the entire nanosheet (Fig. 2e), suggesting highly uniform crystalline quality across the entire nanosheet. The XRD data indicates that the diffraction peaks can be indexed to cubic phase $\gamma$-Cul, which has a space group F-43 m with lattice parameters of $a=b=c=6.034 \AA$ (JCPDS no. 76-0207) (Fig. 2f). A strong diffraction peak of the (111) plane suggests that the as-grown $\gamma$-Cul nanosheets tend to be preferentially oriented along the (111) direction. Additionally, the stability of $\gamma$-Cul nanosheets was also studied. Figure S3 shows the OM images and Raman spectra of freshly prepared Cul nanosheets and those obtained after a 5 days aging under ambient conditions. The nearly unchanged peak position and weakly reduced peak intensity indicate no significant degradation of the PVD-derived Cul nanosheets.

The synthesized $\mathrm{Y}$-Cul nanosheets are further characterized using SEM and TEM studies (Fig. 3). A typical SEM image reveals the well-faceted triangular nanosheets deposited on $\mathrm{SiO}_{2} / \mathrm{Si}$ substrate (Fig. 3a). The SEM-EDX spectroscopy demonstrates that the nanosheets consist of $\mathrm{Cu}$ and I elements (the exhibited $\mathrm{O}$ and Si elements are from the $\mathrm{SiO}_{2}$ substrate), with the atomic ratio of $\mathrm{Cu}$ and I approximately 1 (Fig. 3b). Figure 3c shows the lowresolution TEM (LR-TEM) image of a typical $\gamma$-Cul nanosheet, where well-faced triangle shape of the nanosheet is clearly identified. The high-resolution TEM (HR-TEM) image of the nanosheet shows well-resolved lattice planes with a periodic atomic arrangement, confirming a single-crystalline nature of the resulted $2 \mathrm{D}$ nanosheets. The lattice spacing of 0.34 and $0.20 \mathrm{~nm}$ can be assigned to the 1/3(422) and (220) planes of cubic structure (Fig. $3 d$ ), respectively. The corresponding SAED pattern (inset of Fig. 3d) shows six-fold rotational symmetry for the diffraction spots, which can be indexed to the cubic-blend structure of $\gamma$-Cul along the [111] zone axis. The single set of diffraction spots further demonstrates the single crystalline nature of the $\gamma$-Cul nanosheets.

Furthermore, we also found that the $\gamma$-Cul nanosheets can be readily grown on $2 \mathrm{D}$ transition metal dichalcogenides (e.g., $2 \mathrm{D}$ $\mathrm{WSe}_{2}, 2 \mathrm{D}-\mathrm{WS}_{2}$ ) to form vertical heterostructures by using a twostep PVD progress. Firstly, we grew the single-crystal $\mathrm{WSe}_{2}\left(\mathrm{WS}_{2}\right)$ nanosheets on the $\mathrm{SiO}_{2} / \mathrm{Si}$ substrate using a PVD method. ${ }^{24,47,48}$ Then, we directly grew the Cul nanosheets on the as-grown 2D$\mathrm{WSe}_{2}\left(\mathrm{WS}_{2}\right)$ substrate (more details are described in the Methods). Interestingly, the $\mathrm{\gamma}$-Cul nanosheets can readily nucleate and grow on the top surface of $\mathrm{WSe}_{2}\left(\mathrm{WS}_{2}\right)$ nanosheets, forming the $\gamma$-Cul/ $W e_{2}\left(\gamma-C u l / W_{2}\right)$ vertical vdWHs (Fig. 4a-c). Figure 4a shows the OM image of the $\gamma$-Cul/WSe $e_{2}$ vertical heterostructures, where the green and light gray domains are the $\gamma$-Cul nanosheets, and the purple domains are the underlying $\mathrm{WSe}_{2}$ nanosheets. Figure $4 \mathrm{~b}, \mathrm{c}$ show optical images of the representative $\gamma$-Cul/ $\mathrm{WSe}_{2}$ and $\gamma$-Cul/ $\mathrm{WS}_{2}$ vertical heterostructures. It is noted that $\mathrm{\gamma}-\mathrm{Cul} / \mathrm{WSe}_{2}$ and $\gamma$ $\mathrm{Cul} / \mathrm{WS}_{2}$ heterostructures apparently show a parallel orientation between the $\gamma$-Cul hexagonal domains and the underlying TMDs, suggesting a preferred van der Waals epitaxial relationship. ${ }^{30}$ Figure $4 d-f$ shows an optical image of a $\mathrm{\gamma}$-Cul/ $\mathrm{WSe}_{2}$ vertical heterostructure, and the corresponding Raman mappings at $122 \mathrm{~cm}^{-1}$ (for $\mathrm{y}^{-} \mathrm{Cul}$ ) and $248 \mathrm{~cm}^{-1}$ (for WSe $\mathrm{C}_{2}$. The Raman studies clearly confirm the formation of vertical heterostructures. Besides, similar $\gamma$-Cul/WS 2 heterostructure have also clearly confirm the formation of vertical heterostructures (Fig. $4 \mathrm{~g}-\mathrm{i})$. Photoluminescence $(\mathrm{PL})$ spectra on the resulted $\gamma-\mathrm{Cu} / \mathrm{WSe}_{2}$ and $\gamma-\mathrm{Cul} / \mathrm{WS}_{2}$ heterostructures were shown in Fig. $4 \mathrm{j}, \mathrm{k}$. The results indicate that the $\mathrm{WS}_{2}$ and $\mathrm{WS}_{2}$ monolayers exhibit strong PL emission with a dominant emission peak locating at 769 and $630 \mathrm{~nm}$, respectively, whereas both $\gamma$-Cul/WSe $e_{2}$ and $\gamma$-Cul/WS 2 regions show apparent PL quenching, with essentially no detectable PL. The observed quenching of the PL in the heterostructure may be attributed to 

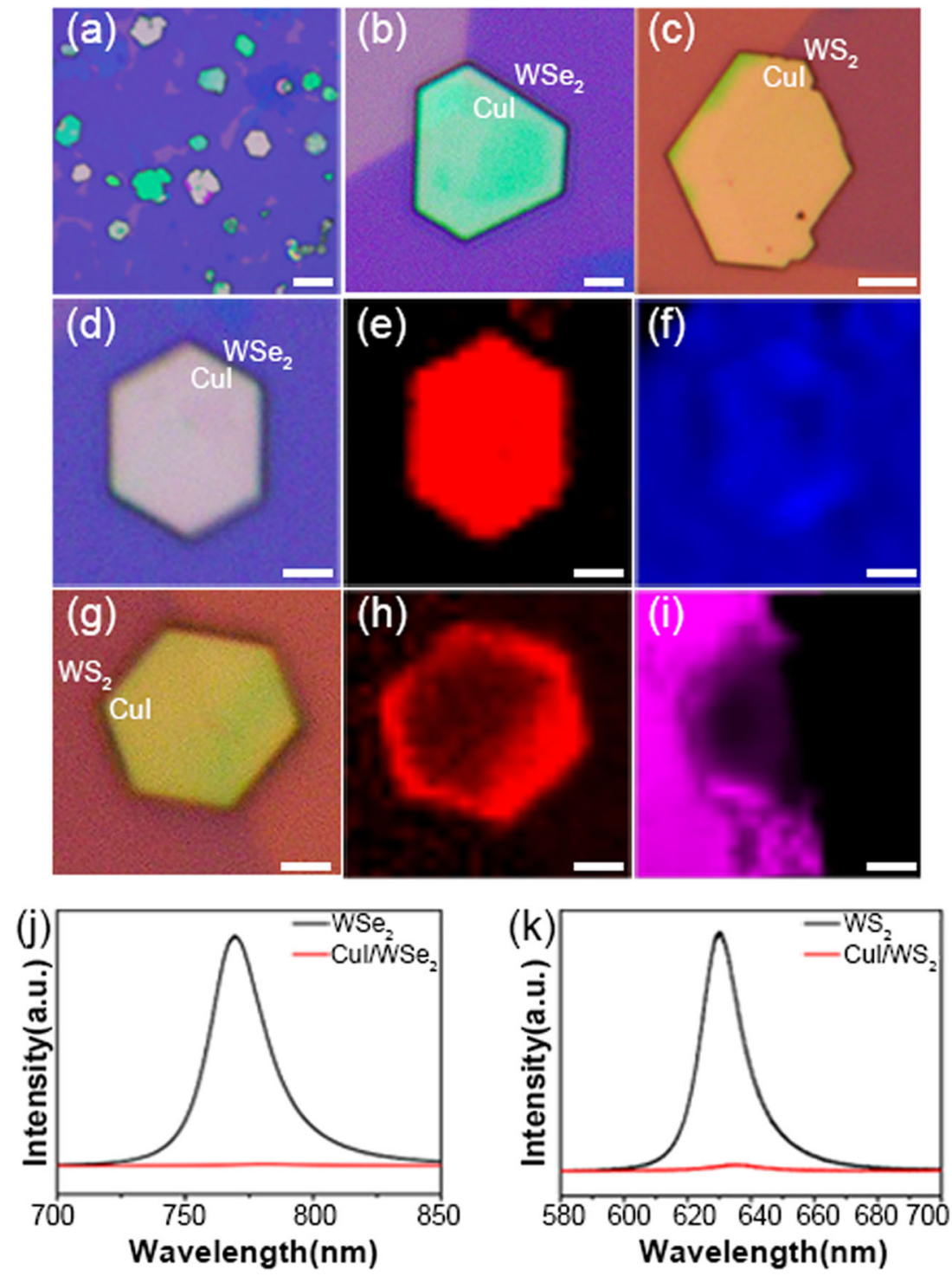

Fig. $4 \mathrm{OM}$, Raman, and PL characterizations of the $\gamma$-Cul/WSe $e_{2}$ and $\gamma$-Cul/WS $\mathrm{W}_{2}$ heterostructures. a OM image of the $\gamma-C u l / W S e_{2}$ heterostructures on the $\mathrm{SiO}_{2} / \mathrm{Si}$ substrate. Scale bar, $10 \mu \mathrm{m}$. b, c High magnification OM image of the representative $\gamma$-Cul/WSe 2 and $\gamma$-Cul/WS vertical heterostructures. Scale bar, 5 and $2 \mu \mathrm{m}$, respectively. d OM image of a typical $\gamma$-Cul/WSe $\mathrm{W}_{2}$ vertical heterostructure used for Raman characterization. Scale bar, $2 \mu \mathrm{m}$. e, $\mathbf{f}$ Raman intensity mappings at 122 and $248 \mathrm{~cm}^{-1}$, corresponding to the characteristic Raman peak of Cul and $\mathrm{WSe}_{2}$, respectively. Scale bar, $2 \mu \mathrm{m}$. g OM image of a typical $\gamma$-Cul/WS 2 vertical heterostructure used for Raman characterization. Scale bar,

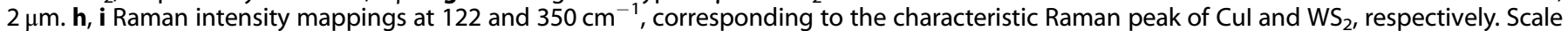
bar, $2 \mu \mathrm{m}$. j PL spectra observed from bare $\mathrm{WSe}_{2}$ region (black curve) and Cul/WSe $\mathrm{W}_{2}$ heterostructure region (red curve). $\mathbf{k} \mathrm{PL}$ spectra observed from bare $\mathrm{WS}_{2}$ region (black curve) and Cul/WS $\mathrm{WS}_{2}$ heterostructure region (red curve).

the spontaneous separation of charge carriers in the junction region. ${ }^{49,50}$

We have further studied the electronic properties of the $\gamma$-Cul nanosheets and $\mathrm{\gamma}$-Cul/ $\mathrm{WS}_{2}$ vertical heterostructure (Fig. 5). The insets of Fig. $5 \mathrm{a}, \mathrm{b}$ show optical images of two $\gamma$-Cul nanosheets devices. The current-voltage $(I-V)$ characteristic curves at room temperature show the linear and symmetric relationship, indicating that the good ohmic contacts are formed (Fig. 5a, b). The resulting $\gamma$-Cul nanosheets show a relatively low resistivity about $0.28 \Omega \mathrm{cm}$ for the $230 \mathrm{~nm}$ thick nanosheets and $0.57 \Omega \mathrm{cm}$ for the $43 \mathrm{~nm}$ nanosheets. The low resistivity of the $\mathrm{\gamma}$-Cul nanosheets may be largely attributed to copper vacancy, acting as an electron acceptor that creates holes in the valence band. ${ }^{51}$ Because $\gamma$-Cul is reported to be a p-type semiconductor, $\mathrm{WSe}_{2}$ is an p-type semiconductor, and $\mathrm{WS}_{2}$ is an n-type semiconductor, ${ }^{47,51,52}$ the $\gamma^{-}$
$\mathrm{Cul} / \mathrm{WSe}_{2}$ vertical heterostructure may be formed a $\mathrm{p}-\mathrm{p}$ junction whereas the $\mathrm{\gamma}$-Cul/WS $\mathrm{WS}_{2}$ vertical heterostructure is expected to behavior as a $\mathrm{p}-\mathrm{n}$ diode. Figure $\mathrm{S} 4$ shows the band alignment and band diagram of $\mathrm{Cul}_{1} \mathrm{WS}_{2}$, and $\mathrm{WSe}_{2}$ across the heterostructure. At zero bias, the conduction band minimum and valence band maximum of Cul are located at a higher energy than that of $\mathrm{WS}_{2}$, forming a type-II heterostructure. Thus, the electrons on the conduction band transfer from Cul to $\mathrm{WS}_{2}$, while the holes on the valence band transfer from $W_{2}$ to Cul resulting in the efficient charge separation. Indeed, the current-voltage characteristics of a typical $\mathrm{\gamma}$-Cul/WS 2 vertical heterostructure show obvious current rectification behavior with a rectification ratio reaching up to 338 at the bias voltage of $\pm 1.5 \mathrm{~V}$ (Fig. $5 \mathrm{c}$ ), consistent with the expected p-n diode behavior. The inset shows the optical image of a typical device where the Au electrodes are separately made on $\gamma$-Cul and 

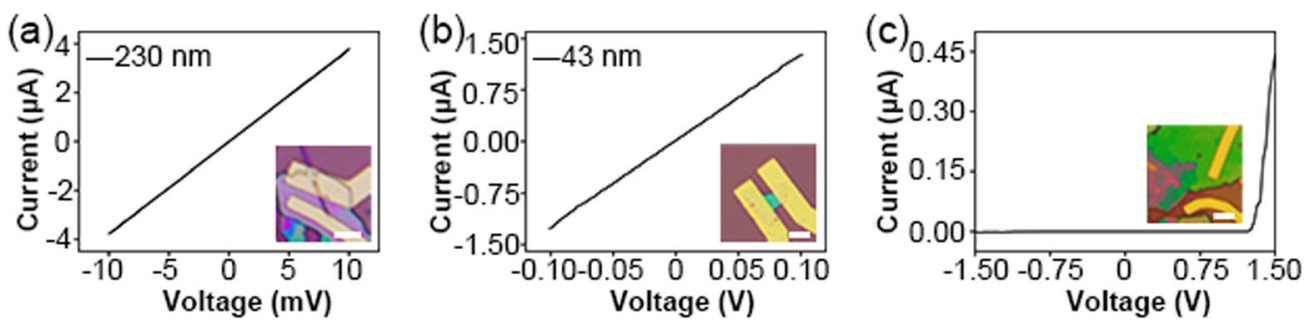

Fig. 5 Electrical characterization of the $\gamma$-Cul nanosheets and $\gamma$-Cul/WS 2 vertical heterostructure. a Current-voltage characteristic of the $\gamma$-Cul nanosheet with the thickness of $230 \mathrm{~nm}$. b Current-voltage characteristic of the $\gamma$-Cul nanosheet with the thickness of 43 nm. c Current-voltage characteristic of the $\gamma$-Cul/WS 2 vertical heterostructure $p-n$ diode. The inset shows the OM image of the corresponding devices. Scale bars, $5 \mu \mathrm{m}$

$\mathrm{WS}_{2}$. We have further deduced the ideality factor using Lamber function: ${ }^{53}$

$I(\mathrm{~V})=\frac{n V_{\mathrm{T}}}{R_{\mathrm{S}}} W_{\mathrm{o}}\left(\frac{I_{\mathrm{S}} R_{\mathrm{S}}}{n V_{\mathrm{T}}} \mathrm{e}^{\left(V+I_{\mathrm{S}} R_{\mathrm{S}}\right) / n V_{\mathrm{T}}}\right)-I_{\mathrm{S}}$,

where $I_{\mathrm{S}}$ is the saturation current, $V_{\mathrm{T}}$ is the thermal voltage $(\approx 0.026 \mathrm{~V}$ at $T=300 \mathrm{~K}), n$ is the ideality factor, and $R_{\mathrm{S}}$ is the series resistance. An ideality factor $n=2.72$ is extracted, which compares well with many other 2D diodes. ${ }^{54,55}$

In summary, we have reported the successful synthesis of ultrathin nanosheets from non-layered $\gamma$-Cul and 2D- $\gamma-\mathrm{Cul} / \mathrm{WSe}_{2}$, $2 \mathrm{D}-\gamma-\mathrm{Cul} / \mathrm{WS}_{2}$ vdWHs on $\mathrm{SiO}_{2} / \mathrm{Si}$ substrate using a facile PVD method. The obtained $\gamma$-Cul nanosheets exhibit triangle shapes and hexagonal shapes with lateral size up to several micrometers and thickness down to $1 \mathrm{~nm}$. The Raman spectra studies show characteristic peak at around $122 \mathrm{~cm}^{-1}$, in accordance with the bulk $\gamma$-Cul. XRD study suggests the obtained nanosheets are pure cubic phase of $\gamma$-Cul crystal with the (111) directions normal to the $\mathrm{SiO}_{2} / \mathrm{Si}$ substrate plane. TEM and SAED studies demonstrate the high crystallinity and single crystal nature of the as-synthesized $\gamma^{-}$ Cul nanosheets. The $\gamma$-Cul/WSe ${ }_{2}$ and $\gamma$-Cul/WS $S_{2}$ vertical heterostructures have been prepared by a two-step PVD progress. The electrical transport studies show that $\gamma$-Cul nanosheets have low revisitivity $\sim 0.3 \Omega \mathrm{cm}$ and $\gamma-C u l / W_{2}$ vertical heterstructure show expected $p-n$ diode behavior. The successful synthesis of 2D nanosheets from non-layered $\gamma$-Cul and van der Waals heterostructures opens a new path way to explore ultrathin 2D structure from other non-layered materials and could enable new opportunities for electronic and optoelectronic devices.

\section{METHODS}

\section{Synthesis of single crystal $\gamma$-Cul nanosheets}

The $y$-Cul nanosheets were grown on $300 \mathrm{~nm} \mathrm{SiO} / 2 \mathrm{Si}$ substrate using a home-built PVD system. Figure S1 shows the illustration of the PVD setup for $y$-Cul growth. Briefly, $0.5 \mathrm{~g}$ Cul powder (Alfa, 99.5\%) in a small ceramic boat was placed in the center of a horizontal tube furnace with 1-inch diameter quartz tube. The $300 \mathrm{~nm} \mathrm{SiO}_{2} / \mathrm{Si}$ substrate $(\sim 1 \mathrm{~cm} \times 3 \mathrm{~cm})$ was placed in another boat downstream about $10 \mathrm{~cm}$ away from the center of the quartz tube. Prior to the growth, the tube was purged with $\sim 400 \mathrm{sccm}$ Ar carrier gas (Rizhen, $99.999 \%$ ) flow for $10 \mathrm{~min}$ at room temperature to remove residual oxygen in the reactor. The furnace was then ramped to the desired growth temperature $360-410^{\circ} \mathrm{C}$, and kept for $10-40$ min under ambient pressure with an argon gas flow of $100-225 \mathrm{sccm}$. Note that the temperature of the growth substrate at the downstream end is $193-234^{\circ} \mathrm{C}$ when the center heating zone is set at $360-410^{\circ} \mathrm{C}$. The growth was terminated by shutting off the power of the furnace, and the system was naturally cooled down to room temperature.

\section{Synthesis of $\mathrm{WSe}_{2}$ and $\mathrm{WS}_{2}$ nanosheets}

$\mathrm{WSe}_{2}$ nanosheets: A ceramic boat loaded with $\mathrm{WSe}_{2}$ powder (Sigma Aldrich, 99.9\%) was placed in the center of the furnace. A $300 \mathrm{~nm} \mathrm{SiO} / 2 \mathrm{Si}$ substrate was placed face-up on the other boat downstream. Before heating, high-purity argon gas was continuously introduced into the quartz tube. The furnace was then heated to $1200^{\circ} \mathrm{C}$ and a constant flow of $100 \mathrm{sccm}$ Ar was used as carrier gas. Note that the temperature of the growth substrate at the downstream end is $782^{\circ} \mathrm{C}$ when the center heating zone is set at $1200^{\circ} \mathrm{C}$. After stabilizing for $8 \mathrm{~min}$, the growth was terminated by shutting off the power of the furnace, and the system was naturally cooled down to room temperature. $\mathrm{WS}_{2}$ nanosheets: Similar to the growth of $\mathrm{WS}_{2}$ nanosheets, a ceramic boat loaded with $\mathrm{WS}_{2}$ powder (Sigma Aldrich, 99.9\%) was placed into a ceramic boat at the center of the furnace. A $300 \mathrm{~nm} \mathrm{SiO} / 2 / \mathrm{Si}$ substrate was placed at the end of the furnace. During the growth of $\mathrm{WSe}_{2}$ nanosheets, the furnace temperature was heated to $1180^{\circ} \mathrm{C}$ in an argon environment and maintained 8 min with a $50 \mathrm{sccm}$ of $\mathrm{Ar}$ as carrier gas. Note that the temperature of the substrate at the down stream end is about $754^{\circ} \mathrm{C}$ when the center heating zone is set at $1180^{\circ} \mathrm{C}$. In the end, the growth was terminated by shutting off the power of the furnace, and the system was naturally reduced to room temperature.

Synthesis of $2 \mathrm{D} \mathrm{Y}-\mathrm{Cul} / \mathrm{WSe}_{2}$ and $\mathrm{\gamma}-\mathrm{Cul} / \mathrm{WS}_{2}$ van der Waals heterostructures

The $2 \mathrm{D} \gamma$-Cul/ $\mathrm{WSe}_{2}\left(\gamma-\mathrm{Cul} / \mathrm{WS}_{2}\right)$ vertical heterostructure was grown in an atmospheric pressure PVD system using Cul powder as the source. A ceramic boat loaded with Cul powder was placed into the heating zone of a quartz tube and the $\mathrm{SiO}_{2} / \mathrm{Si}$ substrate with as grown $\mathrm{WS}_{2}\left(\mathrm{WS}_{2}\right)$ nanosheets was placed at the downstream end of the tube furnace. The system was purged with high-purity Ar gas (Rizhen, 99.999\%) before ramping to the desired growth temperature for the growth of $\gamma$-Cul nanoplates at $450^{\circ} \mathrm{C}$, and maintained for $5 \mathrm{~min}$ with a $150 \mathrm{sccm}$ of $\mathrm{Ar}$ as carrier gas. Note that the temperature of the substrate at the downstream end is at $262{ }^{\circ} \mathrm{C}$ when the center heating zone is set at $450^{\circ} \mathrm{C}$. Finally, the growth was terminated by shutting off the power of the furnace, and the system was naturally cooled down to room temperature.

\section{Sample characterizations}

The $\gamma$-Cul nanosheets grown on the $\mathrm{SiO}_{2} / \mathrm{Si}$ substrate were first characterized using an optical microscope (DP27, OLYMPUS). The thickness of $\gamma$-Cul nanosheets was determined using an atomic force microscope (Bioscope system, BRUCKER). The crystal structure of the sample was studied using a X-ray diffraction spectrometer (XRD-6100, SHIMADZU). The morphology and element composition of the obtained $\gamma$-Cul nanosheets were characterized using a SEM microscope ( $($ IGMA HD, ZEISS) equipped with an EDX system. TEM characterization was performed using a TEM microscope (JEM-2100F, JEOL), operating at $200 \mathrm{kV}$. Raman spectra from the $\gamma$-Cul nanosheets and the $\gamma$-Cul/WSe ${ }_{2}\left(W_{2}\right)$ heterostructures were collected using a confocal microscopic spectrometer (invia-reflex, Renishaw) with $532 \mathrm{~nm}$ laser as the excitation source.

\section{Device fabrication and characterization}

The $\gamma$-Cul nanosheet devices and $p-n$ junction devices were fabricated using e-beam lithography and thermal evaporation of $150 \mathrm{~nm} \mathrm{Au}$ electrode. The electrical measurements were done at room temperature under dark condition.

Data availability

The data related to the findings of this work are available from the corresponding author on reasonable request. 


\section{ACKNOWLEDGEMENTS}

We acknowledge the financial support from the National Natural Science Foundation of China (No. 61528403 and No. 21673070) and the Fundamental Research Funds of the Central Universities (No. 531107051078).

\section{AUTHOR CONTRIBUTIONS}

X.D.D., J.H., and X.D. designed the research. K.Y. performed the synthesis and characterization of the samples. P.C. fabricated and measured the device. Z.Z. prepared the $\mathrm{WS}_{2}$ and $\mathrm{WSe}_{2}$ sample. All authors contributed to experiment research and commented on the manuscript.

\section{ADDITIONAL INFORMATION}

Supplementary information accompanies the paper on the npj 2D Materials and Applications website (https://doi.org/10.1038/s41699-018-0058-2).

Competing interests: The authors declare no competing interest.

Publisher's note: Springer Nature remains neutral with regard to jurisdictional claims in published maps and institutional affiliations.

\section{REFERENCES}

1. Novoselov, K. S. et al. Electric field effect in atomically thin carbon films. Science 306, 666-669 (2004).

2. Jin, C., Lin, F., Suenaga, K. \& lijima, S. Fabrication of a freestanding boron nitride single layer and its defect assignments. Phys. Rev. Lett. 102, 195505 (2009).

3. Wang, X., Feng, H., Wu, Y. \& Jiao, L. Controlled synthesis of highly crystalline $\mathrm{MoS}_{2}$ flakes by chemical vapor deposition. J. Am. Chem. Soc. 135, 5304-5307 (2013).

4. Song, J.-G. et al. Layer controlled wafer-scale and conformal synthesis of tungesten disulfide nanosheets using atomic layer deposition. ACS Nano 7, 11333-11340 (2013).

5. Chen, Y.-Z. et al. Ultrafast and low temperature synthesis of highly crystalline and patternable few-layers tungsten diselenide by laser irradiation assisted selenization process. ACS Nano 9, 4346-4353 (2015).

6. Alsaif, M. M. et al. Tunable plasmon resonances in two-dimensional molybdenum oxide nanoflakes. Adv. Mater. 26, 3931-3937 (2014).

7. Yan, J. et al. Tungsten oxide single crystal nanosheets for enhanced multichannel solar light harvesting. Adv. Mater. 27, 1580-1586 (2015).

8. Wang, Y., Sun, Y.-Y., Zhang, S., Lu, T.-M. \& Shi, J. Band gap engineering of a soft inorganic compound $\mathrm{Pbl}_{2}$ by incommensurate van der Waals epitaxy. Appl. Phys. Lett. 108, 013105 (2016).

9. $\mathrm{Ai}, \mathrm{R}$. Q. et al. Growth of single-crystalline cadmium iodide nanoplates, $\mathrm{Cdl}_{2} / \mathrm{MoS}_{2}$ $\left(\mathrm{WS}_{2}, \mathrm{WSe}_{2}\right.$ ) van der Waals heterostructures, and patterned arrays. ACS Nano 11, 3413-3419 (2017)

10. $\mathrm{Li}$, J. et al. Synthesis of $2 \mathrm{D}$ layered $\mathrm{Bil}_{3}$ nanoplates, $\mathrm{Bil}_{3} / \mathrm{WSe}_{2}$ van der Waals heterostructures and their electronic, optoelectronic properties. Small 13, 1701034 (2017).

11. Huang, B. et al. Layer-dependent ferromagnetism in a van der Waals crystal down to the monolayer limit. Nature 546, 270-273 (2017).

12. Liu, X. et al. High performance field-effect transistor based on multilayer tungsten disulfide. ACS Nano 8, 10396-10402 (2014).

13. Huang, Y. et al. Designing the shape evolution of $\mathrm{SnSe}_{2}$ nanosheets and their optoelectronic properties. Nanoscale 7, 17375-17380 (2015).

14. Zhou, J. et al. Controlled synthesis of high-quality monolayered a- $\ln _{2} \mathrm{Se}_{3}$ via physical vapor deposition. Nano Lett. 15, 6400-6405 (2015).

15. Chow, W. L. et al. High mobility $2 D$ palladium diselenide field-effect transistors with tunable ambipolar characteristics. Adv. Mater. 29, 1602969 (2017).

16. Zhou, C. et al. Carrier type control of $\mathrm{WSe}_{2}$ field-effect transistors by thickness modulation and $\mathrm{MoO}_{3}$ layer doping. Adv. Funct. Mater. 26, 4223-4230 (2016).

17. Ren, X. et al. Environmentally robust black phosphorus nanosheets in solution: application for self-powered photodetector. Adv. Funct. Mater. 27, 1606834 (2017).

18. Cheng, L. et al. Ultrathin $\mathrm{WS}_{2}$ nanoflakes as a high-performance electrocatalyst for the hydrogen evolution reaction. Angew. Chem. Int. Ed. 53, 7860-7863 (2014).

19. Chuang, H. J. et al. Low-resistance $2 \mathrm{D} / 2 \mathrm{D}$ Ohmic contacts: a universal approach to high-performance $\mathrm{WSe}_{2}, \mathrm{MoS}_{2}$, and $\mathrm{MoSe}_{2}$ transistors. Nano Lett. 16, 1896-1902 (2016).

20. Movva, H. C. P. et al. High-mobility holes in dual-gated $\mathrm{WSe}_{2}$ field-effect transistors. ACS Nano 9, 10402-10410 (2015).

21. Zhao, H. et al. Interlayer interactions in anisotropic atomically thin rhenium diselenide. Nano Res. 8, 3651-3661 (2015).
22. $\mathrm{Yu}, \mathrm{Z}$. et al. Analyzing the carrier mobility in transition-metal dichalcogenide $\mathrm{MoS}_{2}$ field-effect transistors. Adv. Funct. Mater. 27, 1604093 (2017)

23. Radisavljevic, B., Radenovic, A., Brivio, J., Giacometti, V. \& Kis, A. Single-layer $\mathrm{MoS}_{2}$ transistors. Nat. Nanotechnol. 6, 147-150 (2011).

24. Georgiou, T. et al. Vertical field-effect transistor based on graphene-WS $\mathrm{S}_{2}$ heterostructures for flexible and transparent electronics. Nat. Nanotechnol. 8, 100-103 (2013).

25. $\mathrm{Hu}, \mathrm{P}$. et al. Highly responsive ultrathin $\mathrm{GaS}$ nanosheet photodectors on grid and flexible substrates. Nano Lett. 13, 1649-1654 (2013).

26. Cheng, R. et al. Electroluminescence and photocurrent generation from atomically sharp $\mathrm{WSe}_{2} / \mathrm{MoS}_{2}$ heterojunction p-n diodes. Nano Lett. 14, 5590-5597 (2014).

27. Murali, K., Dandu, M, \& Majumdar, K. Gate-tunable $\mathrm{WSe}_{2} / \mathrm{SnSe}_{2}$ backward diode with ultrahigh-reverse rectification ratio. ACS Appl. Mater. Interfaces 10, 5657-5664 (2018)

28. Sun, Y. et al. Fabrication of flexible and freestanding zinc chalcogenide single layers. Nat. Commun. 3, 1057 (2012).

29. Acharya, S. et al. A bottom-up approach toward fabrication of ultrathin $\mathrm{PbS}$ sheets. Nano Lett. 13, 409-415 (2013).

30. Zheng, W. et al. Anisotropic growth of nonlayered CdS on $\mathrm{MoS}_{2}$ monolayer for functional vertical heterostructures. Adv. Funct. Mater. 26, 2648-2654 (2016).

31. Wen, Y. et al. Integrated high-performance infrared phototransistor arrays composed of nonlayered PbS-MoS 2 heterostructures with edge contacts. Nano Lett. 16, 6437-6444 (2016).

32. Miyake, S., Hoshino, S. \& Takenaka, T. On the phase transition in cuprous iodide. J. Phys. Soc. Jpn 7, 19-24 (1952).

33. Wang, J., Li, J. \& Li, S.-S. Native p-type transparent conductive Cul via intrinsic defects. J. Appl. Phys. 110, 054907 (2011).

34. $\mathrm{Xu}, \mathrm{H}$. J. et al. Cul-nanoparticles-catalyzed selective synthesis of phenols, anilines, and ihiophenols from aryl halides in aqueous solution. J. Org. Chem. 76, 2296-2300 (2011).

35. Rochford, L. A. et al. Structural templating in a nonplanar phthalocyanine using single crystal copper iodide. Adv. Mater. Interfaces 2, 1400540 (2015).

36. Kumara, G. R. A., Konno, A., Shiratsuchi, K., Tsukahara, J. \& Tennakone, K. Dyesensitized solid-state solar cells: use of crystal growth inhibitors for deposition of the hole collector. Chem. Mater. 14, 954-955 (2002).

37. Yum, J. H., Chen, P., Gratzel, M. \& Nazeeruddin, M. K. Recent developments in solid-state dye-sensitized solar cells. ChemSusChem 1, 699-707 (2008).

38. Sun, W. et al. Room-temperature and solution-processed copper iodide as the hole transport layer for inverted planar perovskite solar cells. Nanoscale 8, 15954-15960 (2016).

39. Christians, J. A., Fung, R. C. \& Kamat, P. V. An inorganic hole conductor for organolead halide perovskite solar cells. improved hole conductivity with copper iodide. J. Am. Chem. Soc. 136, 758-764 (2014).

40. Sharma, B. \& Rabinal, M. K. Ambient synthesis and optoelectronic properties of copper iodide semiconductor nanoparticles. J. Alloy Compd. 556, 198-202 (2013).

41. Meng, L.-R. et al. Synthesis of luminescent cubic phase one-dimensional Cul nanostructures in solution. Cryst. Growth Des. 10, 3387-3390 (2010).

42. Kang, H., Liu, R., Chen, K., Zheng, Y. \& Xu, Z. Electrodeposition and optical properties of highly oriented $\gamma$-Cul thin films. Electrochim. Acta 55, 8121-8125 (2010).

43. Xu, Y., Chen, D., Jiao, X. \& Ba, L. PEG-assisted fabrication of single-crystalline Cul nanosheets: a general route to two-dimensional nanostructured materials. J. Phys. Chem. C 111, 6-9 (2007).

44. Zhang, B. et al. Morphogenesis of Cul nanocrystals by a TSA-assisted photochemical route: synthesis, optical properties, and growth mechanism. Eur. J. Inorg. Chem. 2009, 1376-1384 (2009).

45. Burns, G., Dacol, F. H., Shafer, M. W. \& Alben, R. The Raman spectra of the superionic conductor Cul in its three phases. Solid State Commun. 24, 753-757 (1977).

46. Kaushik, D. K., Selvaraj, M., Ramu, S. \& Subrahmanyam, A. Thermal evaporated copper iodide (Cul) thin films: a note on the disorder evaluated through the temperature dependent electrical properties. Sol. Energy Mater. Sol. Cells 165, 52-58 (2017)

47. Zhou, H. et al. Large area growth and electrical properties of p-type $\mathrm{WSe}_{2}$ atomic layers. Nano Lett. 15, 709-713 (2014).

48. Elías, A. L. et al. Controlled synthesis and transfer of large-area $\mathrm{WS}_{2}$ sheets: from single layer to few layers. ACS Nano 7, 5235-5242 (2013).

49. $\mathrm{Li}, \mathrm{X}$. et al. Two-dimensional $\mathrm{GaSe} / \mathrm{MoSe}_{2}$ misfit bilayer heterojunctions by van der Waals epitaxy. Sci. Adv. 2, e1501882 (2016).

50. Lee, C. H. et al. Atomically thin $\mathrm{p}-\mathrm{n}$ junctions with van der Waals heterointerfaces. Nat. Nanotechnol. 9, 676-681 (2014).

51. Yamada, N., Ino, R. \& Ninomiya, Y. Truly transparent p-type $\gamma$-Cul thin films with high hole mobility. Chem. Mater. 28, 4971-4981 (2016). 
52. Duan, X. et al. Lateral epitaxial growth of two-dimensional layered semiconductor heterojunctions. Nat. Nanotechnol. 9, 1024-1030 (2014).

53. Banwell, T. C. \& Jayakumar, A. Exact analytical solution for current flow through diode with series resistance. Electron. Lett. 36, 291-292 (2000).

54. Deng, Y. et al. Black phosphorus-monolayer $\mathrm{MoS}_{2}$ van der Waals Heterojunction p-n Diode. ACS Nano 8, 8292-8299 (2014).

55. $\mathrm{Yu}, \mathrm{W}$. J. et al. Vertically stacked multi-heterostructures of layered materials for logic transistors and complementary inverters. Nat. Mater. 12, 246-252 (2013).

Open Access This article is licensed under a Creative Commons Attribution 4.0 International License, which permits use, sharing, adaptation, distribution and reproduction in any medium or format, as long as you give appropriate credit to the original author(s) and the source, provide a link to the Creative Commons license, and indicate if changes were made. The images or other third party material in this article are included in the article's Creative Commons license, unless indicated otherwise in a credit line to the material. If material is not included in the article's Creative Commons license and your intended use is not permitted by statutory regulation or exceeds the permitted use, you will need to obtain permission directly from the copyright holder. To view a copy of this license, visit http://creativecommons. org/licenses/by/4.0/.

(c) The Author(s) 2018 